\title{
Immediate conditional hyperbolicity in dynamical systems
}

\author{
JOSEPH ROSENBLATT AND RICHARD SWANSON \\ Department of Mathematics, The Ohio State University, Columbus, Ohio 43210, \\ USA; Department of Mathematical Sciences, Montana State University, Bozeman, \\ Montana 59717, USA
}

(Received 1 November 1982 and revised 11 April 1983)

\begin{abstract}
For many diffeomorphisms of a compact manifold $X$, eventual conditional hyperbolicity implies immediate conditional hyperbolicity in some (possibly new) Finsler structures. That is, if $A$ and $B$ are vector bundle isomorphisms over the mapping $f$ of the base $X$, such that $\lim _{n \rightarrow \infty}\left\|A_{x}^{n}\right\|\left\|B_{x}^{n}\right\|=0$ uniformly on $X$, then there exist new norms for $A$ and $B$ such that $\left|A_{x}\right|\left|B_{x}\right| \leq \delta<1$ uniformly on $X$, whenever the mapping $f$ satisfies the condition that there exist infinitely many $N \geq 1$ such that any $f^{N}$-invariant Borel measure is $f$-invariant. For example, this condition on $f$ holds if any one of the following conditions holds: (1) $f$ is periodic; (2) $f$ is periodic on its non-wandering set; (3) $f$ has a finite non-wandering set (for example, $f$ is a Morse-Smale diffeomorphism); (4) $f$ is an almost periodic mapping of a connected base $X$; (5) $f$ is a mapping of the circle with no periodic points; or (6) $f$ and all its powers are uniquely ergodic. We consider various types of eventually conditionally hyperbolic systems and describe sufficient conditions on $f$ to have immediate conditional hyperbolicity of these systems in some new Finsler structures. Thus, for a sizable class of dynamical systems, we settle, in the affirmative, a question raised by Hirsch, Pugh, and Shub.
\end{abstract}

\section{0 . Introduction}

In [6], the results of a series of papers by the authors on the structure of hyperbolic sets for diffeomorphisms are gathered together and presented as a unified theory. One technical aspect of [6] concerns the definition of hyperbolicity, whether immediate normal hyperbolicity or eventual normal hyperbolicity is the primary structural property to consider. While immediate normal hyperbolicity is simpler to use, eventual normal hyperbolicity turned out to be all that is needed to prove the main theorems in [6]. It was, and still is, unresolved whether these two notions are identical, up to a change of Finslers or norms. In this paper, we study this question in an abstract setting and prove that it is frequently the case that these two types of hyperbolicity are the same. In fact, just knowing that certain spectral properties hold for the base mapping $f$ is enough to guarantee the equivalence of these two notions for all conditionally hyperbolic systems over the base mapping $f$. We have divided the presentation into three sections. In the first two sections, the abstract problem and most of the main theorems are formulated and proved. This section 
requires no real knowledge of differentiable dynamical systems and is mostly an application of ergodic theory and topological dynamics to our main problem. The third section contains our results for differentiable dynamical systems and for the relationship between immediate and eventual normal hyperbolicity of sets for diffeomorphisms. We hope that the general formulation of the problem as we present it in $\$ 1$ will attract some people to studying this problem who might not have otherwise considered it, and that this will lead to more complete theorems and examples than we have been able to provide.

\section{Conditionally contractive systems}

Let $X$ be a compact Hausdorff space and let $E$ be a continuous vector bundle over the base $X$ with fibres $E_{x}$ isomorphic to some fixed Banach space $B$. Let $f: X \rightarrow X$ be a homeomorphism and let $A: E \rightarrow E$ be some vector bundle isomorphisms over $f$. That is, there is a continuous mapping $A$ on $X$ such that each $A_{x}, x \in X$, is a linear isomorphism from the fibre $E_{x}$ onto the fibre $E_{f(x)}$. As usual, for each $n \geq 0$, the composition

will be denoted by $A_{x}^{n+1}$.

$$
A_{f^{n}(x)} A_{f^{n-1}(x)} \ldots A_{x}
$$

We assume that the vector bundle is given a Finsler structure; that is, a continuous choice of norms $\|\cdot\|_{x}$ on the fibres $E_{x}$ is given. If $u_{x} \in E_{x}$, then $\left\|u_{x}\right\|_{x}$ will usually be denoted by $\left\|u_{x}\right\|$.

(1.1) Definition. The system $(f, A,\|\cdot\|)$ is contractive if there exist some $n \geq 1$ and $0<\delta<1$ such that for all $x \in X$ and $u_{x} \in E_{x}$, we have

$$
\left\|A_{x}^{n} u_{x}\right\| \leq \delta\left\|u_{x}\right\| .
$$

The system $(f, A,\|\cdot\|)$ is immediately contractive if there exists $0<\delta<1$ such that for all $x \in X$ and $u_{x} \in E_{x}$, we have

$$
\left\|A_{x} u_{x}\right\| \leq \delta\left\|u_{x}\right\|
$$

This first proposition is easy to prove.

(1.2) Proposition. The following are equivalent:

(a) the system $(f, A,\|\cdot\|)$ is contractive;

(b) $\lim _{n \rightarrow \infty}\left\|A_{x}^{n}\right\|=0$ uniformly on $X$;

(c) there exist constants $C>0,0<\delta<1$, such that

$$
\left\|A_{x}^{n} u_{x}\right\| \leq C \delta^{n}\left\|u_{x}\right\| \quad \text { for all } x \in X \text { and } u_{x} \in E_{x} .
$$

This shows that whether or not a system $(f, A,\|\cdot\|)$ is contractive does not depend on the choice of Finsler structure, while the system's being immediately contractive in addition does depend on the Finsler structure. As was pointed out by J. Mather [9] in the context of differentiable dynamics, given a contractive system $(f, A,\|\cdot\|)$, one can always choose a new Finsler $|\cdot|$ for which the $\operatorname{system}(f, A,|\cdot|)$ is immediately contractive. To do this, define

$$
r_{A}(x)=\limsup _{n \rightarrow \infty}\left\|A_{x}^{n}\right\|^{1 / n} \quad \text { for all } x \in X
$$


Then when $(f, A,\|\cdot\|)$ is contractive as in $(1.2 c)$, we have

$$
r_{A}(x) \leq \delta \quad \text { for } x \in X
$$

Choose any $\lambda, \delta<\lambda<1$, and define a new Finsler by

$$
\left|u_{x}\right|=\sum_{n=1}^{\infty}\left\|A_{x}^{n} u_{x}\right\| / \lambda^{n} .
$$

Because $r_{A} \leq \delta<\lambda$, this series converges uniformly and defines a new Finsler on the bundle $E$. Notice that $\lambda$ can be chosen arbitrarily close to $\delta$ and that, by compactness of $X$, the sums defining the new Finsler can be chosen to be finite sums.

We are primarily interested in two-variable versions of this type of renorming problem. We assume that we have two systems $\left(f, X, E, A,\|\cdot\|_{A}\right)$ and $\left(f, X, F, B,\|\cdot\|_{B}\right)$ over two vector bundles $E$ and $F$ with the same base $X$. The fibres in $E$ and $F$ are not necessarily isomorphic and there are different Finslers given for the two vector bundles.

(1.3) Definition. The system $(f, A, B)$ is conditionally contractive if there exist $n \geq 1$ and $0<\delta<1$ such that for all $x \in X$ and $u_{x} \in E_{x}, v_{x} \in F_{x}$, we have

$$
\left\|A_{x}^{n} u_{x}\right\|\left\|B_{x}^{n} v_{x}\right\| \leq \delta\left\|u_{x}\right\|\left\|v_{x}\right\|
$$

The system $(f, A, B)$ is immediately conditionally contractive if there exists $0<\delta<1$ such that for all $x \in X$ and $u_{x} \in E_{x}, v_{x} \in F_{x}$, we have

$$
\left\|A_{x} u_{x}\right\|\left\|B_{x} v_{x}\right\| \leq \delta\left\|u_{x}\right\|\left\|v_{x}\right\|
$$

Here again, a system $(f, A, B)$ is conditionally contractive if and only if there exist some constants $C>0$ and $0<\delta<1$ such that

$$
\left\|A_{x}^{n}\right\|\left\|B_{x}^{n}\right\| \leq C \delta^{n} \quad \text { for all } x \in X ;
$$

and this property is independent of the Finsler structures on $E$ and $F$ up to choosing a different value of $C$. However, whether the system is immediately conditionally contractive depends strongly on the Finslers which are used. It should be noticed that if $(f, A, B)$ is conditionally contractive, then it need not follow that either $(f, A)$ or $(f, B)$ is contractive.

The problem we wish to solve generally is whether, given a conditionally contractive system $(f, A, B$ ), we can choose new Finslers for $E$ and $F$ for which the system is immediately conditionally contractive. This problem was first considered by Hirsch, Pugh, and Shub in [6] where it arises when studying hyperbolic sets for diffeomorphisms, and they made a few remarks on this general problem; see $\S 3$ for a discussion.

First, we look at a case that is easily handled for all base mappings $f$. Even so, this takes care of many quite complicated dynamical systems.

(1.4) THEOREM. Suppose that the system $(f, A, B)$ is conditionally contractive and that $A$ or $B$ has rank one. Then there are new Finslers for $E$ and $F$ for which $(f, A, B)$ is immediately conditionally contractive.

Proof. Suppose the fibres $F_{x}$ are one-dimensional. For each $x \in X$, choose a vector $e_{x} \in F_{x}$ with $\left\|e_{x}\right\|=1$. While we might not be able to choose $\left(e_{x}\right)$ continuously, the 
functions

$$
x \rightarrow\left\|B_{x}^{n} e_{x}\right\|
$$

are continuous. Choose $C>0$ and $0<\delta<1$ such that

$$
\left\|A_{x}^{n}\right\|\left\|B_{x}^{n}\right\| \leq C \delta^{n} \quad \text { for all } n \geq 1 .
$$

Let $\delta<\lambda<1$. Define a new norm $|\cdot|_{x}$ on $E$ by

$$
\left|u_{x}\right|=\sum_{n=1}^{\infty}\left\|A_{x}^{n} u_{x}\right\|\left\|B_{x}^{n} e_{x}\right\| / \lambda^{n}
$$

for all $u_{x} \in E_{x}$. Since the terms in the sum are continuous, this defines a new Finsler on $E$. For $u_{x} \in E_{x}$ and $v_{x} \in F_{x}$, we have

$$
\begin{aligned}
\left|A_{x} u_{x}\right|\left\|B_{x} v_{x}\right\| & =\sum_{n=1}^{\infty}\left\|A_{f(x)}^{n} A_{x} u_{x}\right\|\left\|B_{f(x)}^{n} e_{f(x)}\right\|\left\|B_{x} v_{x}\right\| / \lambda^{n} \\
& =\sum_{n=1}^{\infty}\left\|A_{x}^{n+1} u_{x}\right\|\left\|B_{f(x)}^{n}\left(B_{x} v_{x}\right)\right\| / \lambda^{n} \\
& =\lambda\left\|v_{x}\right\| \sum_{n=1}^{\infty}\left\|A_{x}^{n+1} u_{x}\right\|\left\|B_{x}^{n+1} e_{x}\right\| / \lambda^{n+1} \\
& \leq \lambda\left|u_{x}\right|\left\|v_{x}\right\| .
\end{aligned}
$$

It is reasonable to try to adapt Mather's construction directly to more general conditionally contractive systems $(f, A, B)$. This requires a solution of the following question.

(1.5) Problem. When do there exist continuous functions $\gamma_{A}>0$ and $\gamma_{B}>0$ on $X$ such that

(a) $\gamma_{A}(f(x))=\gamma_{A}(x), \gamma_{B}(f(x))=\gamma_{B}(x) \quad$ for $x \in X$;

(b) $\gamma_{A}(x) \gamma_{B}(x)<1 \quad$ for $x \in X$;

(c) there exists $N \geq 1$ such that $\left\|A_{x}^{n}\right\| \leq \gamma_{A}^{n}(x)$ for all $n \geq N, x \in X$;

(d) there exists $N \geq 1$ such that $\left\|B_{x}^{n}\right\| \leq \gamma_{B}^{n}(x)$ for all $n \geq N, x \in X$ ?

Suppose that (a)-(d) hold. Then define new Finslers, for $\varepsilon>0$, by

$$
\left|u_{x}\right|=\sum_{n=1}^{\infty}\left\|A_{x}^{n} u_{x}\right\| /\left(\gamma_{A}(x)+\varepsilon\right)^{n}, \quad\left|v_{x}\right|=\sum_{n=1}^{\infty}\left\|B_{x}^{n} v_{x}\right\| /\left(\gamma_{B}^{n}(x)+\varepsilon\right)^{n}
$$

for all $u_{x} \in E_{x}, v_{x} \in F_{x}$. These series converge uniformly on $X$ by (c), (d) and so define Finslers for $E$ and $F$. By (a), we have, for all $u_{x} \in E_{x}, v_{x} \in F_{x}$,

$$
\left|A_{x} u_{x}\right|\left|B_{x} v_{x}\right| \leq\left(\gamma_{A}(x)+\varepsilon\right)\left(\gamma_{B}(x)+\varepsilon\right)\left|u_{x}\right|\left|v_{x}\right| .
$$

By (b), if $\varepsilon>0$ is sufficiently small, the system $(f, A, B)$ is immediately conditionally contractive in the new Finslers $|\cdot|$.

The next proposition is an application of the technique above. We get this proposition in $\S 2$ also, but not by this argument.

(1.6) Proposition. Suppose $f$ is periodic and the system $(f, A, B)$ is conditionally contractive. Then there are new Finslers for $E$ and $F$ for which $(f, A, B)$ is immediately conditionally contractive. 
Proof. Suppose $f^{N+1}(x)=x$ for all $x \in X$. Then

$$
A_{x}^{(N+1) m}=\left(A_{f}^{N}(x) \ldots A_{x}\right)^{m}=\left(A_{x}^{N+1}\right)^{m} .
$$

It is easy to see from this that

$$
\delta_{A}(x)=\lim _{k \rightarrow \infty}\left\|A_{x}^{k}\right\|^{1 / k}
$$

exists for all $x \in X$. Indeed, $\left(\delta_{A}(x)\right)^{N+1}$ is just the usual spectral radius of $A_{x}^{N+1}$ : $E_{x} \rightarrow E_{x}$. Also, since the sequence

$$
\left(\left\|\left(A_{x}^{N+1}\right)^{2 m}\right\|^{1 / 2 m}: m \geq 1\right)
$$

decreases to $\delta_{A}^{N+1}(x)$ pointwise on $X$, the function $\delta_{A}$ is upper semicontinuous on $X$. Similarly

$$
\delta_{B}(x)=\lim _{k \rightarrow \infty}\left\|B_{x}^{k}\right\|^{1 / k}
$$

is upper semicontinuous on $X$. Since the $\operatorname{system}(f, A, B)$ is conditionally contractive, there exists $C>0$ and $\delta>0$ such that

$$
\left\|A_{x}^{n}\right\|\left\|B_{x}^{n}\right\| \leq C \delta^{n}
$$

for all $x \in X$ and $n \geq 1$. So

$$
\delta_{A}(x) \delta_{B}(x) \leq \delta<1
$$

for $x \in X$. It is an easy consequence of the compactness of $X$ and the upper semicontinuity of $\delta_{A}$ and $\delta_{B}$ that, for all $\varepsilon>0$, there exist continuous functions $\gamma_{A}^{1}$ and $\gamma_{B}^{1}$ on $X$ such that

$$
\delta_{A} \leq \gamma_{A}^{1}, \quad \delta_{B} \leq \gamma_{B}^{1}, \quad \text { and } \quad \gamma_{A}^{1}(x) \gamma_{B}^{1}(x) \leq \delta+\varepsilon
$$

for all $x \in X$. The functions $\gamma_{A}^{1}$ and $\gamma_{B}^{1}$ may not be $f$-invariant, so define

$$
\gamma_{A}^{2}(x)=\left(\prod_{n=0}^{N+1} \gamma_{A}^{1}\left(f^{n}(x)\right)\right)^{1 / N+1}
$$

and

$$
\gamma_{B}^{2}(x)=\left(\prod_{n=0}^{N+1} \gamma_{B}^{1}\left(f^{n}(x)\right)\right)^{1 / N+1}
$$

for all $x \in X$. Then $\gamma_{A}^{2}$ and $\gamma_{B}^{2}$ are $f$-invariant. Since $\delta_{A}$ and $\delta_{B}$ are $f$-invariant, we still have

$$
\delta_{A} \leq \gamma_{A}^{2}, \quad \delta_{B} \leq \gamma_{B}^{2}, \quad \text { and } \quad \gamma_{A}^{2} \gamma_{B}^{2} \leq \delta+\varepsilon .
$$

Now let $\gamma_{A}=\gamma_{A}^{2}+\varepsilon$ and $\gamma_{B}=\gamma_{B}^{2}+\varepsilon$. For $\varepsilon>0$ sufficiently small, $\gamma_{A}$ and $\gamma_{B}$ satisfy (a) and (d) of (1.5).

The class of periodic maps $f$ seems to be the only one to which this argument easily applies. But it is reasonable to hope that for some dynamical systems in which the underlying mapping is ergodic, a technique along these lines might give renorming theorems; however, there is a serious technical difficulty in this approach. Assume that $\mu$ is some $f$-invariant Borel probability measure on $X$. Such measures always exist, but not always with the support of $\mu$ being all of $X$. Of course, even in this case, the mapping $f$ may not be ergodic for $\mu$ (e.g. classical dynamical systems, see 
[1]). In any event, Kingman [7] has shown that a.e. [ $\mu] x \in X$, the orbit spectral radius $\lim _{n \rightarrow \infty}\left\|A_{x}^{n}\right\|^{1 / n}$ exists. This is the reason that for any system $(f, A)$, there exists at least one point $x \in X$ such that $\lim _{n \rightarrow \infty}\left\|A_{x}^{n}\right\|^{1 / n}$ exists. If $(X, \mu, f)$ is ergodic, then $\lim _{n \rightarrow \infty}\left\|A_{x}^{n}\right\|^{1 / n}$ is also constant a.e. $[\mu]$ (see [7] and [11]). Assume that $(X, \mu, f)$ is ergodic and that the support of $\mu$, denoted supp $(\mu)$, is all of $X$. Let $r_{A}$ be the constant with $r_{A}=\lim _{n \rightarrow \infty}\left\|A_{x}^{n}\right\|^{1 / n}$ a.e. [ $\mu$ ]. Let $\lambda_{A}>r_{A}$ and define a new norm for $u_{x} \in E_{x}$ by

$$
\left|u_{x}\right|=\sum_{n=1}^{\infty}\left\|A_{x}^{n} u_{x}\right\| / \lambda_{A}^{n}
$$

Although $|\cdot|$ is defined a.e. $[\mu]$, it does not necessarily extend to a continuous Finsler structure on $E$. To do this, we would need a stronger convergence property than that given by the subadditive ergodic theorem.

(1.7) Definition. Let $(X, \mu, f)$ be ergodic with $\operatorname{supp}(\mu)=X$. Then the system $(f, A)$ has a uniform spectral radius if, for all $\varepsilon>0$, there exists $N \geq 1$ such that

$$
\left\|A_{x}^{n}\right\| \leq\left(r_{A}+\varepsilon\right)^{n} \quad \text { for all } n \geq N \text { and } x \in X .
$$

It is easy to see that the system $(f, A)$ has a uniform spectral radius if and only if, for all $\varepsilon>0$, there is a Finsler structure on $E$ with $\left|A_{x}\right| \leq r_{A}+\varepsilon$. By considering the case that $A$ has rank one, one can see that $f$ must be uniquely ergodic in order that every system $(f, A)$ should have a uniform spectral radius. However, it does not appear likely that unique ergodicity of $f$ is enough to guarantee that every system $(f, A)$ has a uniform spectral radius because the counterexamples in [4] show that there is no uniform subadditive ergodic theorem for uniquely ergodic mappings $f$ of the base $X$. At least, if $f$ is uniquely ergodic and $A$ is a continuous direct sum of rank one bundles, then $(f, A)$ does have a uniform spectral radius. Using Finslers as in (5), the next proposition follows directly from definition 1.7.

(1.8) Proposition. Let $(f, A, B)$ be a conditionally contractive system and suppose that both $(f, A)$ and $(f, B)$ have uniform spectral radius. Then there are new Finslers for $E$ and $F$ for which $(f, A, B)$ is immediately conditionally contractive.

There is another type of renorming problem which also appears in [6]. This type of conditional hyperbolicity is different from the usual notion of hyperbolicity of a diffeomorphism.

(1.9) Definition. Let $(f, A, B, C)$ be a system of three different vector bundle isomorphisms. The system is conditionally hyperbolic if there exists $0<\delta<1$ and $n \geq 1$ such that for all $x \in X, u_{x} \in E_{x}, u_{f^{n}(x)} \in E_{f^{n}(x)}, v_{f^{n}(x)} \in F_{f^{n}(x)}$, and $w_{x} \in G_{x}$,

$$
\left\|A_{x}^{n} u_{x}\right\|\left\|\left(B^{-1}\right)_{f^{n}(x)}^{n} v_{f^{n}(x)}\right\| \leq \delta\left\|u_{x}\right\|\left\|v_{f^{n}(x)}\right\|
$$

and

$$
\left\|\left(A^{-1}\right)_{f^{n}(x)}^{n_{(x)}} u_{f^{n}(x)}\right\|\left\|C_{x}^{n} w_{x}\right\| \leq \delta\left\|u_{f^{n}(x)}\right\|\left\|w_{x}\right\| .
$$

The system is immediately conditionally hyperbolic if there exists $0<\delta<1$ such that for all $x \in X, u_{f(x)} \in E_{f(x)}, v_{f(x)} \in F_{f(x)}$, and $w_{x} \in G_{x}$,

$$
\left\|A_{x} u_{x}\right\|\left\|\left(B^{-1}\right)_{f(x)} v_{f(x)}\right\| \leq \delta\left\|u_{x}\right\|\left\|v_{f(x)}\right\|
$$


and

$$
\left\|\left(A^{-1}\right)_{f(x)} u_{f(x)}\right\|\left\|C_{x} w_{x}\right\| \leq \delta\left\|u_{f(x)}\right\|\left\|w_{x}\right\| .
$$

We are again most interested in whether, given a conditionally hyperbolic system ( $f, A, B, C$ ), there exist new Finsler structures for $E, F$, and $G$ which makes the system immediately conditionally hyperbolic. This question poses even more difficulties in general than the one we have been considering. For instance, we do not know an analogue of theorem 1.4 when $B$ and $C$ are both rank one. In the case that $f$ is ergodic with respect to a probability measure $\mu$ on $X$ having full support, there is an analogue to proposition 1.8. We need lemma 1.10 first.

(1.10) LEMMA. Suppose fis ergodic with respect to a regular Borel probability measure $\mu$ with full support. Let $(f, A)$ be a system over the base $X$. Then for a.e. $[\mu] x \in X$, we have

$$
\lim _{n \rightarrow \infty}\left\|A_{x}^{n}\right\|^{1 / n}=\lim _{n \rightarrow \infty}\left\|A_{f}^{n}(x)\right\|^{1 / n}
$$

and

$$
\lim _{n \rightarrow \infty}\left\|A_{x}^{n}\right\|^{1 / n} \cdot \lim _{n \rightarrow \infty}\left\|A_{x}^{-n}\right\|^{1 / n} \geq 1 .
$$

Proof. We know that there are constants $\alpha, \beta$, and $\gamma$ with

$$
\alpha=\lim _{n \rightarrow \infty}\left\|A_{x}^{n}\right\|^{1 / n}, \quad \beta=\lim _{n \rightarrow \infty}\left\|A_{x}^{-n}\right\|^{1 / n} \quad \text { and } \quad \gamma=\lim _{n \rightarrow \infty}\left\|A_{f}^{n}(x)\right\|^{1 / n}
$$

for a.e. $[\mu] x \in X$. This is seen by Kingman [7] using the isomorphisms $A, A^{-1}$, and $A^{*}$. Here $A$ and $A^{-1}$ both act on the original vector bundle $E$ while $A^{*}$ acts on the dual bundle $E^{*}$ with fibres $E_{x}^{*}$, the dual space of $E_{x}$; moreover $A^{-1}$ and $A^{*}$ both act over the mapping $f^{-1}$ of the base $X$.

There must exist a compact set $K \subset X, \mu(K)>0$, such that the functions $\left(\left\|A_{x}^{n}\right\|^{1 / n}\right.$ : $n \geq 1$ ) converge uniformly to $\alpha$ on $K$. Hence, for all $\varepsilon>0$, there exists $N \geq 1$ such that if $n \geq N$ and $x \in K$, then

$$
\left\|A_{x}^{n}\right\|^{1 / n} \in(\alpha(1-\varepsilon), \alpha(1+\varepsilon)) .
$$

Since $\mu(K)>0$ and $f$ is ergodic, for a.e. $x \in X$, infinitely many iterates $f^{-m}(x)$ are in $K$. Choose such an $x_{0} \in K$ and an $m \geq N$. Then

$$
\left\|A_{f^{-m}\left(x_{0}\right)}^{m}\right\|^{1 / m} \in(\alpha(1-\varepsilon), \alpha(1+\varepsilon)) .
$$

This shows in particular that $\gamma \in(\alpha(1-\varepsilon), \alpha(1+\varepsilon))$ by letting $m \rightarrow \infty$ through a subsequence of whole numbers depending on $x_{0} \in K$ as above, but an $x_{0}$ which also satisfies

$$
\lim _{n \rightarrow \infty}\left\|A_{f}^{n-n}\left(x_{0}\right)\right\|^{1 / n}=\gamma
$$

So $\alpha=\gamma$. Moreover, we have

$$
1 \leq\left\|A_{f}^{m}{ }^{m}\left(x_{0}\right)\right\|\left\|A_{x_{0}}^{-m}\right\|
$$

since $A_{f}^{m-m}\left(x_{0}\right) A_{x_{0}}^{-m}$ is the identity on the fibre $E_{x_{0}}$. Hence

$$
1 \leq \alpha(1+\varepsilon)\left\|A_{x_{0}}^{-m}\right\|^{1 / m} \text {. }
$$


Since this is true for a.e. $[\mu] x_{0} \in K$ and infinitely many $m \geq n$ depending on $x_{0}$, letting $m \rightarrow \infty$ for a suitable $x_{0}$ shows that $1 \leq \alpha(1+\varepsilon) \beta$. Letting $\varepsilon \rightarrow 0$, we see that $1 \leq \alpha \beta$.

Remark. It may seem somewhat surprising that

$$
\lim _{n \rightarrow \infty}\left\|A_{f^{-n}(x)}^{n}\right\|^{1 / n}=\lim _{n \rightarrow \infty}\left\|A_{x}^{n}\right\|^{1 / n}
$$

a.e. [ $\mu$ ]. However, it should be realized that if $k_{n} / n \rightarrow \infty$ as $n \rightarrow \infty$, then $\lim _{n \rightarrow \infty}$ $\left\|A_{f}^{n-k_{n(x)}}\right\|^{1 / n}$ does not exist a.e. $[\mu]$ in general. Take the case where $E$ has rank one and consider the corresponding statement as an additive statement as discussed in [3, corollary 3.9].

(1.11) Theorem. Suppose $(X, \mu, f)$ is ergodic with supp $(\mu)=X$. Assume that $(f, A),\left(f, A^{-1}\right),\left(f, B^{-1}\right)$ and $(f, C)$ have uniform spectral radius. If $(f, A, B, C)$ is conditionally hyperbolic, then there are new Finslers for $E, F$, and $G$ for which $(f, A, B, C)$ is immediately conditionally hyperbolic.

Proof. We assume that we have a system $(f, A, B, C)$ which satisfies the condition: there exist $C>0$ and $0<\delta<1$ such that for all $x \in X$ and $n \geq 1$,

$$
\left\|A_{x}^{n}\right\|\left\|B_{f^{n}(x)}^{-n}\right\| \leq C \delta^{n}, \quad\left\|A_{f^{n}(x)}^{-n}\right\|\left\|C_{x}^{n}\right\| \leq C \delta^{n} .
$$

To prove the theorem we show that by increasing $\delta$ slightly and using new Finsler structures on $E, F$, and $G$, we can have (10) with the constant $C=1$.

Let $\alpha, \beta, b$, and $c$ be constants such that $\alpha=\lim _{n \rightarrow \infty}\left\|A_{x}^{n}\right\|^{1 / n}, \beta=\lim _{n \rightarrow \infty}\left\|A_{x}^{-n}\right\|^{1 / n}$, $b=\lim _{n \rightarrow \infty}\left\|B_{x}^{-n}\right\|^{1 / n}$, and $c=\lim _{n \rightarrow \infty}\left\|C_{x}^{n}\right\|^{1 / n}$ for all $x \in X_{0}$ where $\mu\left(X_{0}\right)=1$. By (10), $\alpha b \leq \delta$ and $\beta c \leq \delta$. Choose $\varepsilon>0$ small enough that

$$
(\alpha+\varepsilon)(b+\varepsilon)<1 \text { and }(\beta+\varepsilon)(c+\varepsilon)<1 .
$$

Define Finslers on $E, F$, and $G$ by

$$
\begin{gathered}
\left|u_{x}\right|=\sum_{n=0}^{\infty}\left\|A_{x}^{n} u_{x}\right\| /(\alpha+\varepsilon)^{n}+\sum_{n=1}^{\infty}\left\|\left(A^{-1}\right)_{x}^{n} u_{x}\right\| /(\beta+\varepsilon)^{n}, \\
\left|v_{x}\right|=\sum_{n=1}^{\infty}\left\|B_{x}^{-n} v_{x}\right\| /(b+\varepsilon)^{n}, \quad\left|w_{x}\right|=\sum_{n=1}^{\infty}\left\|C_{x}^{n} w_{x}\right\| /(c+\varepsilon)^{n},
\end{gathered}
$$

for all $x \in X, u_{x} \in E_{x}, v_{x} \in F_{x}$, and $w_{x} \in G_{x}$. As in theorem (1.8), these series converge uniformly over the base $X$. For all $x \in X$,

Also, we claim that

$$
\left|B_{x}^{-1}\right| \leq b+\varepsilon, \quad\left|C_{x}\right| \leq c+\varepsilon .
$$

$$
\left|A_{x}\right| \leq \alpha+\varepsilon \text { and }\left|A_{x}^{-1}\right| \leq \beta+\varepsilon .
$$

Once this is established, we have the system $(f, A, B, C)$ immediately conditionally hyperbolic in the new Finslers because of the choice of $\varepsilon>0$ above.

Fix $x \in X$ and $u_{x} \in E_{x}$. Then we see that

$$
\begin{aligned}
\left|A_{x} u_{x}\right|= & \sum_{n=1}^{\infty}\left\|A_{x}^{n} u_{x}\right\| /(\alpha+\varepsilon)^{n-1}+\left\|u_{x}\right\| /(\beta+\varepsilon)+\sum_{n=1}^{\infty}\left\|\left(A^{-1}\right)_{x}^{n} u_{x}\right\| /(\beta+\varepsilon)^{n+1} \\
= & (\alpha+\varepsilon) \sum_{n=0}^{\infty}\left\|A_{x}^{n} u_{x}\right\| /(\alpha+\varepsilon)^{n}+(\beta+\varepsilon)^{-1} \sum_{n=1}^{\infty}\left\|\left(A^{-1}\right)_{x}^{n} u_{x}\right\| /(\beta+\varepsilon)^{n} \\
& +\left\|u_{x}\right\|\left((\beta+\varepsilon)^{-1}-(\alpha+\varepsilon)\right) .
\end{aligned}
$$


But lemma 1.10 showed $(\alpha+\varepsilon)(\beta+\varepsilon) \geq \alpha \beta \geq 1$. Hence

$$
\left|A_{x} u_{x}\right| \leq(\alpha+\varepsilon)\left|u_{x}\right|+\left\|u_{x}\right\|\left((\beta+\varepsilon)^{-1}-(\alpha+\varepsilon)\right) \leq(\alpha+\varepsilon)\left|u_{x}\right| .
$$

Similarly, if $x \in X$ and $u_{x} \in E_{x}$,

$$
\left|A_{x}^{-1} u_{x}\right| \leq(\beta+\varepsilon)\left|u_{x}\right|
$$

\section{Renorming and invariant measures}

We turn now to a related construction of new Finsler structures from old ones that has much greater flexibility in general and can be used in situations that we have not treated so far. Suppose that $\|\cdot\|$ is a Finsler norm for the vector bundle $E$, and let $A$ be a vector bundle isomorphism over $f$. An obvious way to attach new norms to $E$ which are sensitive to the behaviour of $\left\|A_{x}^{n}\right\|$ is to choose some continuous functions $\left\{a_{n}(x): n=1, \ldots, N\right\}$ on $X, 0<a_{n}(x)<\infty$ for all $x \in X, n=1, \ldots, N$, and then define, for $u_{x} \in E_{x}$,

$$
\left|u_{x}\right|=\sum_{n=1}^{n} a_{n}(x)\left\|A_{x}^{n} u_{x}\right\| .
$$

Since the series used in all the previous constructions of new Finslers may be taken a posteriori to be finite sums, we see that the above definitions are direct generalizations of the previous construction. It turns out that, assuming that the underlying homeomorphism $f$ has certain spectral properties, then for any conditionally contractive system $(f, A, B)$, there are suitable choices of the coefficients above such that one has new Finslers for which the system is immediately conditionally contractive.

Let us denote the operator of composition with $f$ by $T_{f}: C(X) \rightarrow C(X)$ given by $T_{f}(h)=h \circ f$ for $h \in C(X)$. We need to consider $T$ on $C(X)$ and also its dual on $M(X)=C(X)^{*}$, the space of regular Borel measures on $X$ with total variation norm. If $T_{f}^{*}: M(X) \rightarrow M(X)$ is the dual of $T_{f}$, then

$$
T_{f}^{*} \mu(A)=\mu\left(f^{-1} A\right)
$$

for all Borel sets $A \subset X$ and $\mu \in M(X)$. In the remainder of this section, we will be particularly interested in the point spectrum of $T_{f}^{*}$, a specific property of this spectrum being that which allows us to carry out the constructions to follow. For the sake of discussing the spectrum of $T_{f}$ and $T_{f}^{*}$, complex scalars $\mathbb{C}$ will be used. Recall that $\lambda \in \mathbb{C}$ is in the spectrum $\sigma(S)$ of an operator $S$ if $\lambda I-S$ is not invertible. Since $T_{f}-1 \circ T_{f}=I$, it is easy to see that $\overline{\sigma\left(T_{f}\right)}=\sigma\left(T_{f}^{*}\right)$ is a closed subset of the unit circle $T^{1}=\{\lambda \in \mathbb{C}:|\lambda|=1\}$. The point spectrum $\sigma_{p}(S)$ of the operator $S$ is the set of eigenvalues. The residual spectrum $\sigma_{r}(S)$ consists of all $\lambda \in \sigma(S) \backslash \sigma_{p}(S)$ for which the range of $\lambda I-S$ is not dense, and the continuous spectrum $\sigma_{c}(S)=\sigma(S) \backslash\left(\sigma_{p}(S) \cup\right.$ $\left.\sigma_{r}(S)\right)$.

(2.1) Definition. A homeomorphism $f: X \rightarrow X$ is cyclotomic if there exists $N \geq 1$ such that for each $n \geq N$, the point spectrum $\sigma_{p}\left(T_{f}^{*}\right)$ contains some $n$ 'th-root of unity $\lambda \neq 1$.

In one form or the other, the negation of this spectral property is the one that we need; the mapping $f$ is non-cyclotomic if there exist infinitely many $n$ such that the only $n$ 'th-root of unity in $\sigma_{p}\left(T_{f}^{*}\right)$ is 1 . For instance, we will see that if $f$ has periodic 
points of all orders, then $f$ is cyclotomic. However, $f$ can have no periodic points and still be cyclotomic. The following proposition is worth observing here.

(2.2) Proposition. For any homeomorphism $f: X \rightarrow X, \sigma_{p}\left(T_{f}^{*}\right)=\sigma_{p}\left(T_{f}\right) \cup \sigma_{r}\left(T_{f}\right)$. Proof. If $\lambda \in \sigma_{p}\left(T_{f}^{*}\right)$, then there exists a measure $\mu \in M(X), \mu \neq 0$, such that $T_{f}^{*} \mu=\lambda \mu$. Then for all $h \in C(X)$,

$$
\int_{X}\left(\lambda h-T_{f}(h)\right) d \mu=0 .
$$

Hence, either $\lambda I-T_{f}$ is not one-to-one and $\lambda \in \sigma_{p}\left(T_{f}\right)$, or $\lambda I-T_{f}$ is one-to-one, but does not have dense range (because its range is annihilated by $\mu$ ) and $\lambda \in \sigma_{r}\left(T_{f}\right)$.

Conversely, if $\lambda \in \sigma_{r}\left(T_{f}\right)$, then there exists $\mu \neq 0$ in $M(X)$ such that

$$
\int_{X}\left(\lambda h-T_{f}(h)\right) d \mu=0
$$

for all $h \in C(X)$, and so $\lambda \in \sigma_{p}\left(T_{f}^{*}\right)$. If $\lambda \in \sigma_{p}\left(T_{f}\right)$, there exists $h_{0} \neq 0$ in $C(X)$ with $T_{f}\left(h_{0}\right)=\bar{\lambda} h_{0}$. Let $M \subset X$ be a minimal closed $f$-invariant subset of $X$. Let $\mu$ be any $f$-invariant measure with supp $(\mu)=M$. Then let

$$
\nu(L)=\int_{L} h_{0} d \mu
$$

for all Borel sets $L \subset X$. This gives $\nu \in M(X)$ satisfying $T_{f}^{*}(\nu)=\lambda \nu$. Also if $h_{0}(x) \neq 0$ for some $x \in M$, then $\nu \neq 0$. We see then that $\lambda \in \sigma_{p}\left(T_{f}^{*}\right)$ or $h_{0}=0$ on all minimal closed $f$-invariant subsets of $X$. The latter is impossible since $h_{0} \neq 0$. Indeed, for any $x \in X$, there exists a minimal closed $f$-invariant set $M, y \in M$, and a net $\left(n_{\alpha}\right)$ of whole numbers with $\lim _{\alpha} f^{n_{\alpha}}(x)=y$. Applying this to the equation $h_{0} \circ f=\bar{\lambda} h_{0}$ shows that

$$
0=h_{0}(y)=\lim _{\alpha} \lambda^{-n_{\alpha}} h_{0}(x) .
$$

Since $|\bar{\lambda}|=1$, this forces $h_{0}(x)=0$, and so $h_{0}=0$. Hence, $\sigma_{p}\left(T_{f}\right) \subset \sigma_{p}\left(T_{f}^{*}\right)$.

The actual usefulness of the class of non-cyclotomic mappings comes from the next result.

(2.3) Proposition. Fix $N \geq 1$. The following are equivalent:

(a) there exists $0 \neq \mu \in M(X)$ such that $\sum_{n=0}^{N} T_{f^{n}}^{*} \mu=0$;

(b) there exists $0 \neq \mu \in M(X)$ such that $T_{f^{N+1}}^{*} \mu=\mu$ while $T_{f}^{*} \mu \neq \mu$;

(c) there exist $\nu \neq 0$ and some $(N+1)$ 'st-root of unity $\lambda \neq 1$ with $T_{f}^{*} \nu=\lambda \nu$;

(d) the linear subspace $\left\{\sum_{n=0}^{N} T_{f^{n}}(h): h \in C(X)\right\}$ is not dense in $C(X)$.

(2.4) COROLLARY. The mapping $f$ is non-cyclotomic if and only if one of the following two equivalent conditions holds:

(a) for infinitely many $N \geq 1$, any $\mu \in M(X)$, which is $f^{N}$-invariant, must be f-invariant;

(b) for infinitely many $N \geq 1$, the subspace $\left\{\sum_{n=0}^{N} T_{f^{n}}(h): h \in C(X)\right\}$ is dense in $C(X)$. 
Proof of (2.3). The equivalence of (a) and (d) is just the usual duality argument, a subspace $S \subset C(X)$ being not dense if and only if there exists $0 \neq \mu \in M(X)$ with $\int h d \mu=0$ for all $h \in S$.

Suppose that (a) holds. Then

$$
0=\sum_{n=0}^{N} T_{f^{n}}^{*} \mu=\sum_{n=1}^{N+1} T_{f^{n}}^{*} \mu .
$$

So $T_{f^{N+1}}^{*} \mu=\mu$, but $T_{f}^{*} \mu \neq \mu$ since $\mu \neq 0$. So (a) implies (b). On the other hand if $0 \neq \mu \in M(X)$ with $T_{f}^{*} \mu \neq \mu$ and $T_{f^{N+1}}^{*} \mu \neq \mu$, then

$$
0=\mu-T_{f^{N+1}}^{*} \mu=\sum_{n=0}^{N} T_{f^{n}}^{*}\left(\mu-T_{f}^{*} \mu\right) .
$$

Since $\mu-T_{f}^{*} \mu \neq 0$, (b) implies (a). Assume (c). Then

$$
\sum_{n=0}^{N} T_{f^{n}}^{*} \nu=\left(\sum_{n=0}^{N} \lambda^{n}\right) \nu=0 \cdot \nu=0
$$

because $\lambda \neq 0$ and $\lambda$ is an $(N+1)$ 'st-root of unity. Hence, (c) implies (a). Conversely, if (a) holds, then let $\lambda$ be any $(N+1)$ 'st-root of unity. Let

$$
\nu_{\lambda}=\sum_{n=0}^{N} \lambda^{n} T_{f}^{*} \mu
$$

Because $\sum_{n=0}^{N} T_{f^{n}}^{*} \mu=0$, we have $T_{f^{N+1}}^{*} \mu=\mu$. Hence

$$
T_{f}^{*} \nu_{\lambda}=\sum_{n=0}^{N} \lambda^{n} T_{f^{n+1}}^{*} \mu=\bar{\lambda} \sum_{n=0}^{N} \lambda^{n+1} T_{f}^{*+1} \mu=\bar{\lambda} \nu_{\lambda} .
$$

We claim that for some $\lambda \neq 1$, the measure $\nu_{\lambda} \neq 0$. Indeed, suppose all $\nu_{\lambda}=0$. We then have a system of $N+1$ linear equations in the $N+1$ unknowns

$$
\left(T_{f^{n}}^{*} \mu: n=0, \ldots, N\right)
$$

whose coefficients matrix is $\Lambda=\left(\lambda_{0}^{i j}: i, j=0, \ldots, N\right)$ for some primitive $(N+$ 1)'st-root of unity $\lambda_{0}$. Because $\left(\lambda_{0}^{j}: j=0, \ldots, N\right)$ are distinct, the van der Monde determinant det $\Lambda \neq 0$. Hence, solving this system of linear equations would force all $T_{f^{n}}^{*} \mu=0, n=0, \ldots, N$, which is not the case.

We describe the class of non-cyclotomic maps with examples following the next theorem. This theorem is the main result of this section.

(2.5) THEOREM. Let $f: X \rightarrow X$ be a homeomorphism which is non-cyclotomic. If $(f, A, B)$ is a conditionally contractive system, then there are new Finslers for $E$ and $F$ for which $(f, A, B)$ is immediately conditionally contractive.

Proof. Let us fix some $M \geq 2$ such that

$$
\left(\left\|A_{x}^{N}\right\|\left\|B_{x}^{N}\right\|\right)^{1 / N}<\gamma<1
$$

for all $x \in X$ and $N \geq M$. Choose $N \geq M$ and some functions $\left\{a_{n}: n=1, \ldots, N\right\}$ and $\left\{b_{n}: n=1, \ldots, N\right\}$ in $C(X)$. Define new Finslers on $E$ and $F$ by

$$
\left|u_{x}\right|=\sum_{n=1}^{N} a_{n}(x)\left\|A_{x}^{n} u_{x}\right\| \text { and }\left|v_{x}\right|=\sum_{n=1}^{N} b_{n}(x)\left\|B_{x}^{n} v_{x}\right\|
$$

for all $x \in X, u_{x} \in E_{x}$, and $v_{x} \in F_{x}$. We look for criteria on the coefficient functions 
which allow us to have

$$
\left|A_{x} u_{x}\right|\left|B_{x} v_{x}\right| \leq \gamma\left|u_{x}\right|\left|v_{x}\right|
$$

for all $x \in X, u_{x} \in E_{x}$, and $v_{x} \in F_{x}$. Now

$$
\left|A_{x} u_{x}\right|\left|B_{x} v_{x}\right|=\sum_{m=1}^{N} \sum_{n=1}^{N} a_{m}(f(x)) b_{n}(f(x))\left\|A_{x}^{m+1} u_{x}\right\|\left\|B_{x}^{n+1} v_{x}\right\| .
$$

In trying to overestimate this by $\gamma\left|u_{x}\right|\left|v_{x}\right|$, we match terms with the same factor $\left\|A_{x}^{m+1} u_{x}\right\|\left\|B_{x}^{n+1} v_{x}\right\|$ and impose on $\left\{a_{n}\right\}$ and $\left\{b_{n}\right\}$ the appropriate inequalities. Those terms with factors $\left\|A_{x}^{m+1} u_{x}\right\|\left\|B_{x}^{n+1} v_{x}\right\|$, where one of $m$ or $n$ is $N$, are then compared with earlier terms in the double sum representing $\left|u_{x}\right|\left|v_{x}\right|$ in a cyclical fashion. These comparisons give us the following system of inequalities $\left(I_{j k} ; j, k=1, \ldots, N\right)$ as criteria for (11) to hold:

$$
\begin{aligned}
& I_{11}: a_{1}(f(x)) b_{1}(f(x)) \leq \gamma a_{2}(x) b_{2}(x) \\
& I_{12}: a_{1}(f(x)) b_{2}(f(x)) \leq \gamma a_{2}(x) b_{3}(x) \\
& \vdots \\
& I_{1 N-1}: a_{1}(f(x)) b_{N-1}(f(x)) \leq \gamma a_{2}(x) b_{n}(x) \\
& I_{1 N}: a_{1}(f(x)) b_{N}(f(x))\left\|B_{x}^{N+1} v_{x}\right\| \leq \gamma a_{2}(x) b_{1}(x)\left\|B_{x} v_{x}\right\| . \\
& I_{21}: a_{2}(f(x)) b_{1}(f(x)) \leq \gamma a_{3}(x) b_{2}(x) \\
& \vdots \\
& I_{2 N}: a_{2}(f(x)) b_{N}(f(x))\left\|B_{x}^{N+1} v_{x}\right\| \leq \gamma a_{3}(x) b_{1}(x)\left\|B_{x} v_{x}\right\| . \\
& \vdots \\
& I_{N 1}: a_{N}(f(x)) b_{1}(f(x))\left\|A_{x}^{N+1} u_{x}\right\| \leq \gamma a_{1}(x) b_{2}(x)\left\|A_{x} u_{x}\right\| \\
& \vdots \\
& I_{N N}: a_{N}(f(x)) b_{N}(f(x))\left\|A_{x}^{N+1} u_{x}\right\|\left\|B_{x}^{N+1} v_{x}\right\| \leq \gamma a_{1}(x) b_{1}(x)\left\|A_{x} u_{x}\right\|\left\|B_{x} v_{x}\right\| .
\end{aligned}
$$

Now let us impose the condition that $a_{n}(x) b_{n}(x)=\pi_{n}$, a constant, where we choose $\pi_{N}=1$ and all $\pi_{n}, n=1, \ldots, N-1$, such that

$$
\left\|A_{x}^{N}\right\|\left\|B_{x}^{N}\right\|<\gamma \pi_{1}<\gamma^{2} \pi_{2}<\cdots<\gamma^{N-1} \pi_{N-1}<\gamma^{N} \pi_{N} .
$$

This is possible because $\left\|A_{x}^{N}\right\|\left\|B_{x}^{N}\right\|<\gamma^{N}$ uniformly on $X$. Also, this choice makes the inequalities $I_{i i}, i=1, \ldots, N$, hold automatically. Furthermore, the remaining inequalities can now be expressed in terms of just $\left\{a_{n}\right\}$ and $\left\{\pi_{n}\right\}$. Notice that we have now implicitly assumed that all of the functions $a_{n}$ satisfy $a_{n}(x)>0$ for any $x \in X$.

Let $\alpha_{n}(x)=\ln \left(a_{n}(x)\right), n=1, \ldots, N$. Let us consider the inequalities $I_{N i}$, $i=1, \ldots, N-1$, in terms of $\left\{a_{n}\right\}$. Notice that

$$
\left\|A_{x}^{N+1} u_{x}\right\| /\left\|A_{x} u_{x}\right\| \leq\left\|A_{f(x)}^{N}\right\| \quad \text { if } u_{x} \neq 0 .
$$

Also $\gamma \pi_{i+1} / \pi_{i}>1$ for $i=1, \ldots, N-1$. Hence to have $I_{N i}, i=1, \ldots, N-1$, holding on $X$, it would suffice to choose

$$
\alpha_{N}(f(x))-\alpha_{i}(f(x))-\alpha_{1}(x)+\alpha_{i+1}(x)+\ln \left\|A_{f(x)}^{N}\right\|
$$

uniformly close to zero simultaneously for $i=1, \ldots, N-1$. If we do this, then

$$
\alpha_{i}(f(x))-\alpha_{N}(f(x))+\alpha_{1}(x)-\alpha_{i+1}(x)
$$


is uniformly close to $\ln \left\|A_{f(x)}^{N}\right\|$. But $\left\|B_{f(x)}^{N}\right\| / \gamma \pi_{1}<1 /\left\|A_{f(x)}^{N}\right\|$ on $X$ and so

$$
-\ln \left(\left\|B_{f(x)}^{N}\right\| / \gamma \pi_{1}\right)>\ln \left\|A_{f(x)}^{N}\right\| .
$$

This means that we would have

$$
\alpha_{i}(f(x))-\alpha_{N}(f(x))+\alpha_{1}(x)-\alpha_{i+1}(x)+\ln \left(B_{f(x)}^{N} \| / \gamma \pi_{1}\right)
$$

uniformly close to $\ln \left\|A_{f(x)}^{N}\right\|+\ln \left(\left\|B_{f(x)}^{N}\right\| / \gamma \pi_{1}\right)$ which is strictly less than zero on $X$. That is, in satisfying $I_{N i}, t=1, \ldots, N-1$, in this particular fashion, we automatically get $I_{i N}, i=1, \ldots, N-1$, being satisfied too.

Moreover, if expression (12) is uniformly close to zero, then taking these $N-1$ approximations in pairs, we would get for $i, j=1, \ldots, N-1$

$$
\alpha_{i}(f(x))-\alpha_{j}(f(x))-\alpha_{i+1}(x)+\alpha_{j+1}(x)
$$

uniformly close to zero. Taking the exponential of this approximation, we see that we have, for $i, j=1, \ldots, N-1$,

$$
a_{i}(f(x)) a_{j+1}(x) / a_{j}(f(x)) a_{i+1}(x)
$$

uniformly close to one on $X$. Because $\gamma \pi_{i+1} / \pi_{i}>1$ for $i=1, \ldots, N-1$, this means that all the other inequalities $I_{i j}$ are satisfied too.

Hence, it only remains to construct $\alpha_{1}, \ldots, \alpha_{N} \in C(X)$ such that the expressions (12) are uniformly close to zero, and then $a_{i}=\exp \left(\alpha_{i}\right)$ will give the required $a_{1}, \ldots, a_{N} \in C(X), a_{i}>0$ on $X, i=1, \ldots, N$. Abstractly, this is an approximation problem of the following type: we are given a continuous function

$$
h(x)=-\ln \left\|A_{f(x)}^{N}\right\|
$$

defined on $X$; when can we assert that the vector $(h, h, \ldots, h) \in \bigoplus_{n=1}^{N-1} C(X)$ is in the uniform closure of the subset $S \subset \bigoplus_{n=1}^{N-1} C(X)$ which consists of all $(N-1)$-tuples of the form

$$
\left(T_{f} \alpha_{N}-T_{f} \alpha_{1}-\alpha_{1}+\alpha_{2}, \ldots, T_{f} \alpha_{N}-T_{f} \alpha_{N-1}-\alpha_{1}+\alpha_{N}\right)
$$

for some $\alpha_{1}, \ldots, \alpha_{N} \in C(X)$ ? It is easy to check that $S$ is in fact a subspace of $\bigoplus_{n=1}^{N-1} C(X)$. Also the dual space of $\bigoplus_{n=1}^{N-1} C(X)$ is $\bigoplus_{n=1}^{N-1} M(X)$ with the usual coordinate-wise pairing. By duality then, we find that $\alpha_{1}, \ldots, \alpha_{N}$ exist such that (12) is uniformly close to zero if we have for any $\mu_{1}, \ldots, \mu_{N-1} \in M(X)$,

$$
\text { if } 0=\sum_{n=1}^{N-1} \int_{X}\left(T_{f} \alpha_{N}-T_{f} \alpha_{n}-\alpha_{1}+\alpha_{n+1}\right) d \mu_{n} \quad \text { for all } \alpha_{1}, \ldots, \alpha_{N} \in C(X),
$$

then

$$
\mu_{n}=0 \quad \text { for all } n=1, \ldots, N-1 \text {. }
$$

By collecting terms with a common $\alpha_{n}$, we see that condition (15) on $\mu_{1}, \ldots, \mu_{N-1}$ becomes the conditions:

and

$$
\begin{gathered}
T_{f}^{*} \mu_{1}+\cdots+T_{f}^{*} \mu_{N-1}+\mu_{N-1}=0, \\
T_{f}^{*} \mu_{1}+\mu_{1}+\mu_{2}+\cdots+\mu_{N-1}=0,
\end{gathered}
$$

$$
\mu_{n}-T_{f}^{*} \mu_{n+1}=0, \quad n=1, \ldots, N-2 .
$$

By (18), $\mu_{1}=T_{f^{n-1}}^{*} \mu_{n}, n=1, \ldots, N-1$. Hence (16) or (17) says that

$$
\mu_{1}+T_{f}^{*} \mu_{1}+\cdots+T_{f}^{*-1} \mu_{1}=0 \text {. }
$$


We see then that since $f$ is non-cyclotomic, there exists $N \geq M$ such that (19) is only satisfied for $\mu_{1}=0$. But then (18) forces $\mu_{n}=0$ for $n=1, \ldots, N-1$. Therefore, the expression (12) can be made uniformly close to zero for such values of $N$. As we have explained, this finishes the proof.

Remarks. This construction of new Finsler structures can be modified so that if $E$ and $F$ are Hilbert vector bundles to begin with, then the new Finsler structures can also be taken as induced from inner-products. This is true in addition for all the renorming arguments in $\S 1$. Also, the argument shows that when $\left\|A_{x}^{n}\right\|\left\|B_{x}^{n}\right\| \leq C \delta^{n}$ for some $C>0$ and $0<\delta<1$, and if $\gamma>\delta$, then there are new Finslers with $\left|A_{x}\right|\left|B_{x}\right| \leq \gamma$. Again this aspect of the construction above is also common to all the other arguments.

It seems difficult to identify our spectral property with a specific class of dynamical systems. For this reason, we choose only to identify some special classes of homeomorphisms which are non-cyclotomic. Recall that $x \in X$ is non-wandering if for all open sets $V \subset X, x \in V$, there exists some $n \geq 1$ such that $f^{n}(V) \cap V \neq \emptyset$. The set of non-wandering points of $f$ is denoted by $\Omega_{f}$. We say $f$ is strongly-ergodic if each $f^{k}, k=1,2,3, \ldots$, is uniquely ergodic.

(2.6) Proposition. The homeomorphism $f$ is non-cyclotomic if either of the following two conditions hold:

(a) $f$ is periodic when restricted to its non-wandering set;

(b) $f$ is strongly ergodic.

Proof. For (a), let $\mu \neq 0, T_{f^{N+1}}^{*} \mu=\mu$. Then $\mu$ is $f^{N+1}$-invariant and hence $\operatorname{supp}(\mu) \subset \Omega_{f}$. Say $f^{M}=I$ on $\Omega_{f}$. Then if $N=k M, k=1,2,3, \ldots$, any $f^{N+1}$-invariant measure $\mu$ is also $f$-invariant. For (b), there is only one probability measure $\mu_{0} \in M(X)$ which is invariant for any (or all) $f^{k}, k=1,2,3, \ldots$; hence, any $f^{k}$-invariant measure $\mu$ must be $c \mu_{0}$ for some scalar $c$.

Remarks. From (a), any Morse-Smale diffeomorphism of a smooth compact manifold is non-cyclotomic, as is any map with a finite non-wandering set. This includes a generic set of diffeomorphisms of the unit circle; see [10]. Despite this fact and theorem 2.5 , we can not show that when $X=T^{1}$, then any conditionally contractive system $(f, A, B)$ can be given new Finsler structures in which it is immediately conditionally contractive.

(2.7) COROLlary. The homeomorphism $f$ is non-cyclotomic if either of the following two conditions holds:

(a) $X$ is $T^{1}$ and $f$ has no periodic points;

(b) $X$ is connected and $f$ is almost periodic.

Proof. For (a), in [5], it is shown that when a homeomorphism $f$ of the circle $T^{\mathbf{1}}$ has no periodic points, then $f$ is uniquely ergodic. For (b), if $f$ is a homeomorphism of a connected base $X$ and $X$ is the minimal $f$-invariant set, then $X$ is the minimal $f^{k}$-invariant set for all $k=1,2,3, \ldots$. Hence, (b) follows as in [5].

Remark. Corollary 2.7(b) says that if $X$ is a connected compact abelian group with a generator $g$, then $f(x)=g x, x \in X$, is non-cyclotomic. Also, the above results can 
be used when $\Omega_{f}$ is a finite union of minimal closed $f$-invariant sets which are either discrete or on which $f$ is strongly ergodic. For instance, if $f$ is a diffeomorphism of the two sphere which has fixed north and south poles, attracts all other points toward the equator, and acts as irrational rotation on the equator, then $f$ is non-cyclotomic.

(2.8) Example. We show how theorem 2.5 can be applied to provide renorming for a homeomorphism $f$ which is cyclotomic but which merely decomposes into ones that are non-cyclotomic. This example can be generalized, but we do not do this here for brevity's sake. Consider the mapping

$$
f\left(z_{1}, z_{2}\right)=\left(z_{1} z_{2}, z_{2}\right)
$$

for $\left(z_{1}, z_{2}\right) \in T^{2}$. The mapping $f$ is an automorphism of $T^{2}$ and preserves the Haar measure on $T^{2}$, but it is not ergodic because it leaves invariant every circle $T_{w}=$ $\{(z, w): z \in T\}$. Indeed, on $T_{w}, f$ is either periodic or ergodic in the usual Lebesgue measure on $T_{w}$, depending on whether $w$ is a root of unity or not. Also $\left\{T_{z}: z \in T^{1}\right\}$ foliates $T^{2}$ into circles. Now let $(f, A, B)$ be a conditionally contractive system. For each $w_{0} \in T^{1}$, there exist Finslers on the restricted bundles of $E$ and $F$ over each base $T_{w}$ such that the system $(f, A, B)$ is immediately conditionally contractive over the base $T_{w}$. Indeed, if there are constants $C>0$ and $0<\delta<1$ such that

$$
\left\|A_{x}^{n}\right\|\left\|B_{x}^{n}\right\| \leq C \delta^{n}
$$

for all $x \in T^{2}$ then 1.6 and 2.5 show that, for each $w_{0} \in T^{1}$ and $\varepsilon>0$, there are new Finslers such that

$$
\left|A_{x}^{n}\right|\left|B_{x}^{n}\right|<(\delta+\varepsilon)^{n} \quad \text { for all } x \in T_{w_{0}} .
$$

By continuity, we can extend these Finslers to some tubular neighbourhood

$$
T\left(w_{0}, \varepsilon\right)=\bigcup\left\{T_{w}: \operatorname{dist}\left(w, w_{0}\right) \leq \varepsilon\right\}
$$

of the circle $T_{w_{0}}$, if $\varepsilon=\varepsilon\left(w_{0}\right)$ is sufficiently small, and still have with respect to these new Finslers, denoted by $\|\cdot\|_{\left(w_{0}, \varepsilon\right)}$, for all $x \in T_{w}$ with dist $\left(w, w_{0}\right) \leq \varepsilon$,

$$
\left\|A_{x}^{n}\right\|_{\left(w_{0}, \varepsilon\right)}\left\|B_{x}^{n}\right\|_{\left(w_{0}, \varepsilon\right)} \leq(\delta+\varepsilon)^{n}
$$

for all $n \geq 1$. Now the interiors of $\left\{T\left(w_{0}, \varepsilon\right): w_{0} \in T^{1}\right\}$ cover $T^{2}$; so there are a finite number of them, say $\left\{T\left(w_{i}, \varepsilon_{i}\right): i=1, \ldots, m\right\}$, whose interiors cover $T^{2}$. Let $\left\{\phi_{i}: i=1, \ldots, m\right\}$ be a partition of unity on $T^{1}$ which is subordinate to the cover given by the interiors of $\left\{w\right.$ : dist $\left.\left(w, w_{i}\right) \leq \varepsilon_{i}\right\}$. Define new Finslers on $E$ and $F$ by

$$
\left|u_{(z, w)}\right|=\sum_{i=1}^{m} \phi_{i}(w)\left\|u_{(z, w)}\right\|_{\left(w_{i}, \varepsilon_{i}\right)}, \quad\left|v_{(z, w)}\right|=\sum_{i=1}^{m} \phi_{i}(w)\left\|v_{(z, w)}\right\|_{\left(w_{i}, \varepsilon_{i}\right)}
$$

for all $u_{(z, w)} \in E_{(z, w)}, v_{(z, w)} \in F_{(z, w)}$. It is easy to check that in these Finslers $(f, A, B)$ is an immediately conditionally contractive system with

$$
\left|A_{x}^{n}\right|\left|B_{x}^{n}\right| \leq(\delta+\varepsilon)^{n}
$$

for all $x \in T^{2}$ and $n \geq 1$.

There is another special type of conditionally contractive system for which all of our techniques apply. Suppose $(f, A, B)$ is conditionally contractive and $(f, B)$ is contractive. Can we choose new Finsler structures in which $(f, A, B)$ is immediately 
conditionally contractive and simultaneously $(f, B)$ is immediately contractive? Because of the nature of our constructions, this turns out to be generally the case. We have only to assume (or prove!) that there exists an isometry $J$ over $f$ for the vector bundle $E$. We do not know if such an isometry always exists, but at least if $E$ is a Hilbert vector bundle, then $J=A\left(A^{*} A\right)^{-1 / 2}$ will be an isometry in the original Finsler structure if $A^{*}$ is the usual adjoint on $E$.

(2.9) THEOREM. Suppose $f$ is any mapping such that every conditionally contractive system $(f, A, B)$ can be made immediately contractive in Finsler structures for $E$ and $F$ which are linear combinations of $\left(\left\|A_{x}^{n} \cdot\right\|: n \geq 1\right)$ and $\left(\left\|B_{x}^{n} \cdot\right\|: n \geq 1\right)$ respectively. Suppose $(f, A, B)$ is conditionally contractive with $E$ admitting an isometry $J$ over $F$. Then there are new Finslers for $E$ and $F$ in which $(f, A, B)$ is immediately conditionally contractive and $(f, B)$ is immediately contractive.

Proof. Consider the vector bundle isomorphism $A \oplus J$ on the vector bundle $E \oplus E$. Then the system $(f, A \oplus J, B)$ is conditionally contractive. So there exist new Finsler structures on $E \oplus E$ and $F$ in which $(f, A \oplus J, B)$ is immediately conditionally contractive. Moreover, these new Finslers are linear combinations of $\left(\left\|A_{x}^{n} \cdot\right\| \oplus\left\|J_{x}^{n} \cdot\right\|: n \geq 1\right)$ and $\left\|B_{x}^{n} \cdot\right\|$. By dividing the first new Finsler by the sum of its coefficients, and multiplying the second by this same sum, we have new Finslers on $E \oplus E$ and $F$ such that for some $\delta, 0<\delta<1$,

$$
\left(\left|A_{x} u_{x}^{1}\right|+\left|u_{x}^{2}\right|\right) \cdot\left(\left|B_{x} v_{x}\right|\right) \leq \delta\left(\left|u_{x}^{1}\right|+\left|u_{x}^{2}\right|\right) \cdot\left|v_{x}\right|
$$

for all $u_{x}^{1}, u_{x}^{2} \in E_{x}$ and $v_{x} \in F_{x}$. By choosing $u_{x}^{1}$ or $u_{x}^{2}$ to be zero, we see that in these new Finslers $(f, A, B)$ and $(f, B)$ are immediately conditionally contractive.

It is reasonable to ask what the techniques of theorem 2.5 do for conditionally hyperbolic systems $(f, A, B, C)$. We have computed these with as much generality as possible and found that there appears to be an essential breakdown in the technique. Because our results are so partial for this question, we will state what we found without giving a proof.

(2.10) THEOREM. Suppose that for all $m \geq 1$, any $\mu \in M(X)$ which is $f^{2^{m}}$-invariant must also be $f$-invariant. Then if $(f, A, B, C)$ is a conditionally hyperbolic system, there exist new Finsler structures on $E, F$, and $G$ such that $(f, A, B, C)$ is immediately conditionally hyperbolic.

It is still not clear to us what the general resolution of the renorming problem will be. While our positive results provide some hope that every conditionally contractive system is immediately conditionally contractive in some Finsler structure, there are many natural mappings not included in the ones to which our techniques apply. The difficulty in constructing a counter-example is in finding some way to tie the constant $C$, in the estimate

$$
\left\|A_{x}^{n}\right\|\left\|B_{x}^{n}\right\| \leq C \delta^{n} \quad \text { for } n \geq 1,
$$

to some aspect of the system $(f, A, B)$ which is intrinsic to it and not just an artefact of the Finsler structure. Finally, it should also be remarked in looking for a 
counter-example that the class of homeomorphisms which admit a renorming theorem are closed under continuous conjugate equivalence. Moreover, the spectral property that we use for theorem 2.5 is preserved under just Borel conjugate equivalence. Therefore, a homeomorphism may at first appear to be quite unlike any we have handled, and yet be suitably conjugate to some simpler mapping.

\section{Immediate normal hyperbolicity at invariant manifolds}

The renorming problem addressed in the preceding sections was first raised in the context of differentiable dynamics by Hirsch, Pugh, and Shub [6]. Specifically, from the consideration of familiar notions of stability for fixed points and periodic orbits, those authors were led to formulate various types of hyperbolic behaviour at an invariant compact manifold. In that same source, the reader will also find a similar treatment of flows and, more generally, foliations. It is clear that much of the material of $\S \S 1$ and 2 can be extended to flows, but we have not done that here. In this section, we consider only discrete dynamical systems or diffeomorphisms. Instead of considering arbitrary Finslers on the bundles as in the last section, here we restrict our attention to Riemannian structures. Before contrasting the different ways in which normal hyperbolicity can be expressed, we shall briefly discuss the general problem of seeking 'adapted' metrics which display immediate hyperbolicity.

Let $\Lambda \subset M$ denote a hyperbolic set for the self-diffeomorphism $f: M \rightarrow M$. That is, $\Lambda$ is compact, $f(\Lambda)=\Lambda$, and for any Riemannian metric $M$, there are constants $C>0$ and $0<\lambda<1$, and there is a continuous vector bundle splitting $T_{\Lambda} M=$ $E^{+} \oplus E^{-}$of the tangent bundle of $M$ restricted to $\Lambda$ such that for all $n \geq 0$,

$$
\begin{aligned}
\left\|T f^{n} v\right\| \leq C \lambda^{n}\|v\| & \text { if } v \in E^{+}, \\
\left\|T f^{-n} v\right\| \leq C \lambda^{n}\|v\| & \text { if } v \in E^{-} .
\end{aligned}
$$

In the special case that $\Lambda=M$, we say that $f$ is Anosov. Various authors have noted that while the constant $\lambda$ is intrinsic (i.e. there is a smallest usable $\lambda$ independent of the metric), the constant $C$ is not intrinsic. Then, for Anosov diffeomorphisms $f$, Mather [9] proved that one could always find an adapted metric for which $C=1$, but with a possibly larger $\lambda$ still less than 1 . Thus, for some Riemannian metric $|\cdot|$, one could always assume that there was a constant $\lambda, 0<\lambda<1$, such that

$$
\begin{aligned}
|T f v| \leq \lambda|v| & \text { if } v \in E^{+}, \\
\left|T f^{-1} v\right| \leq \lambda|v| & \text { if } v \in E^{-},
\end{aligned}
$$

in place of (20) and (21) above. Mather's proof can be simplified greatly by analyzing the natural action of $f$ on the Riemannian metrics. Denote the original metric by $\gamma$; so

$$
\gamma_{x}\left(v_{x}, v_{x}\right)=\left\|v_{x}\right\|^{2} \quad \text { for all } v_{x} \in E_{x}^{+}
$$

Then we have,

$$
\left(f_{*} \gamma\right)_{x}\left(v_{1}, v_{2}\right)=\gamma_{f(x)}\left(\text { Tf } v_{1}, \text { Tf } v_{2}\right)
$$

is a linear action on the metrics. Conditions (20) and (21) imply that for some $N \geq 1$,

$$
f_{*}^{N} \gamma(v, v)<\gamma(v, v) \quad \text { for all } v \in E^{+} .
$$


That is, $\gamma-f_{*}^{N} \gamma$ is a positive-definite symmetric tensor on $E^{+}$. But

$$
\left(I-f_{*}^{N}\right) \gamma=\left(I-f_{*}\right)\left(\sum_{k=0}^{N-1} f_{*}^{k} \gamma\right),
$$

and therefore, $\gamma^{+}=\sum_{k=0}^{N-1} f_{*}^{k} \gamma$ is a metric on $E^{+}$with

$$
\gamma^{+}(T f v, T f v)<\gamma^{+}(v, v) \quad \text { for all } v \in E^{+} .
$$

A similar argument with $f_{*}^{-1}$ in place of $f_{*}$ yields a metric $\gamma^{-}$on $E^{-}$with

$$
\gamma^{-}(T f v, T f v)<\gamma^{-}(v, v) \quad \text { for all } v \in E^{-} \text {. }
$$

The adapted metric we want is $\gamma=\gamma^{+} \oplus \gamma^{-}$. This proof generalizes to any hyperbolic set $\Lambda \subset M$. Also, obviously $\gamma^{+} \oplus \gamma^{-}$can be continuously extended off $\Lambda$ to all of $M$.

If a hyperbolic set $\Lambda$ is to enjoy any kind of stability under perturbation of $f$, conditions (20) and (21) are not strong enough. A more vigorous type of hyperbolicity is required; the set $\Lambda$ must be normally hyperbolic. We will assume that $\Lambda=P$ is a compact smooth submanifold of $M$ in the sequel. Now suppose that $f: M \rightarrow M$ is a $C^{1}$ diffeomorphism and there is a $T f$-invariant bundle splitting which is continuous,

$$
T_{P} M=T P \oplus N^{s} P \oplus N^{u} P,
$$

of $T M$ over $P$ such that $T P$ is the usual tangent bundle of $P$ and the normal bundle splits $T f$-invariantly as the sum of the bundle $N^{s} P$ and $N^{u} P$. As in [6], we adopt the useful notation $P f=\left.T f\right|_{T P}, N^{s} f=\left.T f\right|_{N^{s} P}$, and $N^{u} f=\left.T f\right|_{N^{u} P}$.

(3.1) Definition. The system $(f, M, P)$ is immediately normally hyperbolic if for some Riemannian metric $\|\cdot\|_{x}$ on $T M$, one has for all $x \in P$,

(a) $\left\|N_{x}^{s} f\right\|<\left\|\left(P_{x} f\right)^{-1}\right\|^{-k}$;

(b) $\left\|P_{x} f\right\|^{k}<\left\|\left(N_{x}^{u} f\right)^{-1}\right\|^{-1}$.

The criterion (a) on the system $(f, M, P)$ says that the normal derivative $N^{s} f$ is so contractive that its growth rate along any given orbit is dominated by the minimal rate of growth of the tangential map Pf along that orbit. A similar interpretation can be made of (b). More picturesquely, in a moving coordinate system along an orbit in $P$, one would observe points near to the orbit either approaching or receding at exponential rates.

(3.2) Definition. The system $(f, M, P)$ is (eventually) normally hyperbolic if for some $N \geq 1$, the system $\left(f^{N}, M, P\right)$ is immediately normally hyperbolic.

The goal of this section is to prove that these two possible notions of normal hyperbolicity are equivalent in a variety of rather general dynamical systems, by using the results of the foregoing sections.

(3.3) Remarks. It should be emphasized that, save for the possible inequivalence of eventual and immediate normal hyperbolicity, no weaker definition can be given without destroying the stability of $P$ under perturbations of the diffeomorphism $f$. This is because of the persistence theorem of $R$. Mañe [8]. Also a very strong version of normal hyperbolicity, called 'absolute' normal hyperbolicity, is described in [6], and has the property that 'eventual' implies 'immediate' in all cases. The reader 
should also bear in mind that the present question concerns, to some extent, a topological invariant, as suggested in the remarks at the end of $\S 2$. Finally, in [6], the broader notion of an $r$-normally hyperbolic system is defined to handle the stability of $C^{r}$-invariant submanifolds. In this section, we confine our attention to the $C^{1}$ case (which presents problems enough!).

(3.4) Definition. We call the invariant manifold $P$ a $\operatorname{sink}$ if $N^{u} P=0$, a source if $N^{s} P=0$, and a saddle if $N^{u} P \neq 0$ and $N^{s} P \neq 0$.

(3.5) Theorem. Suppose that $(f, M, P)$ is normally hyperbolic and $P$ is a sink or a source such that any of the following is satisfied:

(a) $\operatorname{codim} P=1$;

(b) the systems $\left(f, P f^{ \pm 1}\right),\left(f, N^{s} f\right)$ and $\left(f, N^{u} f^{-1}\right)$ have uniform spectral radius; or

(c) $\left.f\right|_{P}$ is non-cyclotomic.

Then $(f, M, P)$ is immediately normally hyperbolic.

Proof. The cases (a), (b), and (c) were all discussed in the last section as situations in which a system $(f, A, B)$ can be rendered immediately contractive by a suitable renorming. This proof proceeds by casting normal hyperbolicity as a contractive hypothesis.

We first need to construct an appropriate contractive system. Assume that $P$ is a sink manifold, the proof for source manifolds being much the same. Pick some Riemannian metric on $T M$ with $T P$ orthogonal to $N P$. Let $E$ be given the product metric. With respect to the given Riemannian structure on $T P$, we form the dual bundle $T^{*} P$ in the dual Finsler structure. Then let $E=T^{*} P \oplus T P$ in the product metric. The isomorphism $P f^{-1}$ induces a dual isomorphism $P^{*} f^{-1}$ on $T^{*} P$ over the base mapping $f$; in the dual norm on $T^{*} P, P_{x}^{*} f^{-n}$ has the same norm as $P_{x} f^{-n}$. We also need some isometry $I$ of $T P$. It is easy to generate such an isometry. Let $T: T P \rightarrow T P$ be an isomorphism which covers $f$. Let $T^{*}$ be the adjoint of $T$ as usual for the Hilbertian Finsler structures. Then $I=T\left(T^{*} T\right)^{-\frac{1}{2}}$ is an isometry in the original Finsler structure on $E$. Let $A=P^{*} f^{-1} \oplus I$. Finally, let $F=N^{s} P$ and $B=N^{s} f$. It is now easy to see that normal hyperbolicity means exactly that the system $(f, A, B)$ is conditionally contractive.

Now applying (a), (b), or (c) and the results of $\$ \S 1$ and 2 , there exist new Riemannian structures in which $(f, A, B)$ is immediately conditionally contractive. Indeed, we know that there is a Riemannian structure on $F$ and a product Riemannian structure on $E$, say with

$$
|(u, v)|^{2}=|u|_{1}^{2}+|v|_{2}^{2}
$$

for all $(u, v)$ in $T^{*} P \oplus T P$, such that for all $\left(u_{x}, v_{x}\right) \in E_{x}$,

$$
\left(\left|P^{*} f^{-1} u_{x}\right|_{1}^{2}+\left|I_{x} v_{x}\right|^{2}\right)\left|B_{x}\right|^{2}<\left|\left(u_{x}, v_{x}\right)\right|^{2} .
$$

Moreover, by adjusting norms by the sum of the coefficients in each of the cases (a), (b), and (c), as in theorem 2.9 , we may assume $\left|I_{x} v_{x}\right|=\left|v_{x}\right|$ for all $v_{x} \in T P_{x}$. Setting $v_{x}=0$, we see that for all $u_{x} \in T^{*} P$,

$$
\left|P^{*} f^{-1} u_{x}\right|_{1}\left|B_{x}\right|<\left|u_{x}\right|_{1}
$$


Let $|\cdot|$ be the Riemannian metric on $T P$ which is dual to $|\cdot|_{1}$ on $T^{*} P$. Then we have

$$
\left|P_{x}^{*} f^{-1}\right|\left|B_{x}\right|<1 \quad \text { for all } x \in X \text {. }
$$

Hence, for all $x \in X$,

$$
\left|N^{s} f\right|<\left|P_{x} f^{-1}\right|^{-1}
$$

Finally, letting $u_{x}=0$ above shows $\left|B_{x}\right|<1$ because $I_{x}$ is an isometry. These conditions together give immediate normal hyperbolicity in the new Riemannian metrics.

Remarks. If $\operatorname{dim} P=1$, then in some new Riemannian structures,

$$
\left|N_{x}^{s} f\right|<\left|\left(P_{x} f\right)^{-1}\right|^{-1} \quad \text { for all } x \in X .
$$

But we do not seem to be able also to guarantee $\left|N_{x}^{s} f\right|<1$.

(3.6) . TheOREM. Suppose that $P$ is a saddle and that $(f, M, P)$ is normally hyperbolic. Assume that one of the following holds:

(a) the systems $\left(f, P f^{ \pm 1}\right),\left(f, N^{s} f\right)$, and $\left(f, N^{u} f^{-1}\right)$ have uniform spectral radius;

(b) for every $m \geq 1$, any $\left(\left.f\right|_{P}\right)^{2^{m}}$-invariant Borel measure on $P$ is $\left.f\right|_{P}$-invariant. Then $(f, M, P)$ is immediately normally hyperbolic.

Proof. We use theorem 1.11 and theorem 2.10 as in the proof of theorem 3.5.

Remark. It should be noticed that this theorem applies to any mapping $f$ which is strongly ergodic on $P$. This includes any irrational rotation of a torus $T^{n}$. Also, $\left.f\right|_{P}$ could be a Morse-Smale diffeomorphism of $P$ which has no periodic points of period $2^{m}, m \geq 1$. It does not seem likely that this extra restriction will prove to be really necessary; it is only that dyadic periodic points are precluded by the hypothesis of theorem 2.10 .

One final remark about the uniform spectral radius condition would be appropriate. Suppose $(f, M)$ is a smooth dynamical system which preserves an underlying Borel probability measure $\mu$ on $M$. Assume $(f, M, \mu)$ is ergodic. In considering the behaviour of $T f$ on the tangent bundle, the operator $(T f)^{*}$ on continuous vector fields $\Gamma(T M)$ has been studied by several authors. For background see [2]. The question discussed in $\S 1$ of which systems $(f, A)$ have a uniform spectral radius, becomes in this context the following problem: there is a number $r_{A}=\lim _{n \rightarrow \infty}\left\|T f_{x}^{n}\right\|^{1 / n}$ which is the same for a.e. $[\mu] x \in M$; when is $r_{A}$ equal to the spectral radius of $T f^{\#}$ ? Here we can show that $T f^{\#}$ has a spectral radius which is

$$
\sup \left\{\lim _{n \rightarrow \infty} \sup \left\|T f_{x}^{n}\right\|^{1 / n}: x \in X\right\} \text {. }
$$

So our question is just this: for which diffeomorphisms is $r_{A}$ at least as large as lim $\sup _{n \rightarrow \infty}\left\|T f_{x}^{n}\right\|^{1 / n}$ for all $x \in X$ ? The answer to this problem would be very helpful for proving renorming theorems as in this paper. It seems that one needs to know much more about exceptional characteristics than is currently known in order to answer this question.

j. Rosenblatt was partially supported by NSF Grant MCS 8201800 


\section{REFERENCES}

[1] V. I. Arnold \& A. Avez. Ergodic Problems of Classical Mechanics. W. A. Benjamin, Inc: New York-Amsterdam, 1968.

[2] C. Chicone \& R. Swanson. Spectral theory for linearizations of dynamical systems. J. Differential Equations 40 No. 2 (1981), 155-167.

[3] A. del Junco \& J. Rosenblatt. Counterexamples in ergodic theory and number theory. Math. Ann. 245 (1979), 185-197.

[4] Y. Derriennic \& U. Krengel. Subadditive mean ergodic theorems. Ergod. Th. \& Dynam. Sys. 1 (1981), 9-32.

[5] H. Furstenberg. Strict ergodicity and transformations of the torus. Amer. J. Math. 83 (1961), 573-601.

[6] M. W. Hirsch, C. C. Pugh \& M. Shub. Invariant Manifolds. Lecture Notes in Math., No. 583. Springer-Verlag: Berlin-Heidelberg-New York, 1977.

[7] J. F. C. Kingman. Subadditive ergodic theory. Ann. Probab. 1 \# 6 (1973), 883-909.

[8] R. Mañe. Persistent manifolds are normally hyperbolic. Bull. Amer. Math. Soc. 80 (1974), 90-91.

[9] J. Mather. Characterization of Anosov diffeomorphisms. Indag. Math. 30 (1968), 479-483.

[10] Z. Nitecki. Differentiable Dynamics. M.I.T. Press: Cambridge, 1971.

[11] V. I. Oseledec. A multiplicative ergodic theorem, Lyapunov characteristic numbers for a dynamical system. Trans. Moscow Math. Soc. 19 (1968), 197-231. (AMS, Providence, 1969.) 